\title{
Perseverative interference with object-in-place scene learning in rhesus monkeys with bilateral ablation of ventrolateral prefrontal cortex
}

\author{
Mark G. Baxter, ${ }^{1}$ Philip G.F. Browning, and Anna S. Mitchell \\ Department of Experimental Psychology, Oxford University, Oxford OX1 3UD, United Kingdom
}

\begin{abstract}
Surgical disconnection of the frontal cortex and inferotemporal cortex severely impairs many aspects of visual learning and memory, including learning of new object-in-place scene memory problems, a monkey model of episodic memory. As part of a study of specialization within prefrontal cortex in visual learning and memory, we tested monkeys with bilateral ablations of ventrolateral prefrontal cortex in object-in-place scene learning. These monkeys were mildly impaired in scene learning relative to their own preoperative performance, similar in severity to that of monkeys with bilateral ablation of orbital prefrontal cortex. An analysis of response types showed that the monkeys with lesions were specifically impaired in responding to negative feedback during learning: The post-operative increase in errors was limited to trials in which the first response to each new problem, made on the basis of trial and error, was incorrect. This perseverative pattern of deficit was not observed in the same analysis of response types in monkeys with bilateral ablations of the orbital prefrontal cortex, who were equally impaired on trials with correct and incorrect first responses. This may represent a specific signature of ventrolateral prefrontal involvement in episodic learning and memory.
\end{abstract}

The prefrontal cortex is important for multiple aspects of memory, decision making, and cognitive control (Passingham 1993; Goldman-Rakic 1996; Miller 2000). Its involvement in aspects of episodic learning and memory can be tested in macaque monkeys by performance in an object-in-place scene learning task (Gaffan 1994). In this task, monkeys encounter discrimination problems in which they must learn which of two foreground objects is correct in a complex background scene. All of the elements of the scene differ from problem to problem, but the locations and identities of all the scene elements are constant for a particular problem. Monkeys learn object-in-place scene problems very rapidly relative to, for example, similar discrimination problems against a constant or neutral background (Gaffan 1994). The learning of object-in-place scene problems is significantly impaired by damage to the fornix (Gaffan 1994; Aggleton et al. 2000) and perirhinal cortex (Easton and Gaffan 2000).

Damage to the prefrontal cortex, or disconnection of the frontal cortex from inferotemporal cortex by means of crossed unilateral lesions, produces a devastating impairment in learning new scene problems (Browning et al. 2005). The impairment caused by frontal-temporal disconnection can be reproduced, in part, by crossed unilateral lesions of ventrolateral prefrontal cortex and inferotemporal cortex (Wilson et al. 2007). These observations are consistent with a role for the prefrontal cortex in episodic memory and with a role for the ventrolateral prefrontal cortex specifically in aspects of episodic memory (Wagner et al. 2001; Petrides et al. 2002).

The aim of the current experiment was to evaluate scene learning in monkeys with bilateral ventrolateral damage, for comparison with previous data from monkeys with frontalinferotemporal disconnection (Browning et al. 2005), disconnection of ventrolateral prefrontal cortex (via a neurotoxic lesion) from inferotemporal cortex (Wilson et al. 2007), or bilateral ab-

\footnotetext{
1 Corresponding author.

Fax 44-1865-310447.

Article is online at http://www.learnmem.org/cgi/doi/10.1101//m.804508. Freely available online through the Learning \& Memory Open Access option.
}

lation of orbital prefrontal cortex (Baxter et al. 2007). Although disconnection of ventrolateral prefrontal cortex from inferotemporal cortex impaired scene learning (Wilson et al. 2007), those lesions included partial involvement of orbital prefrontal cortex and were produced with a neurotoxic lesion method. Thus, the current study provides a direct comparison with cortical ablations, as well as with the data from the neurotoxic lesion method.

In the present study, five monkeys were taught the scene learning task until they could rapidly learn lists of 20 new scene problems in each session. After a preoperative performance test composed of 10 sessions of new scene learning (200 new scene problems total), each monkey received bilateral ablations of ventrolateral prefrontal cortex. An identical performance test was given post-operatively in which a further 200 new scenes were learned. As part of the analysis of these data, we compared performance in scene problems where the first response (made by trial and error) was wrong with problems in which it was correct. This revealed a unique effect in the group of monkeys with bilateral ventrolateral lesions, in that their impairment was only significant for scenes in which the first response was wrong. We present an analysis of their impairment in scene learning and compare it with results from other prefrontal and inferotemporal cortical lesions, in which impairments in scene learning occur irrespective of the outcome of the first response.

\section{Results}

\section{Extent of ventrolateral prefrontal ablations}

The lesion was intended to remove areas $47 / 12$ and 45A (Petrides and Pandya 2002) bilaterally, using sulcal landmarks to guide the extent of the ablation. A schematic of the lesions is shown in Figure 1, and photomicrographs of sections from each of the five ventrolateral prefrontal lesions are shown in Figure 2. The intended region was ablated in each monkey, although the lesions varied slightly in medial-lateral extent. Case VL5 sustained unintended damage to the lateral orbital cortex bilaterally. 


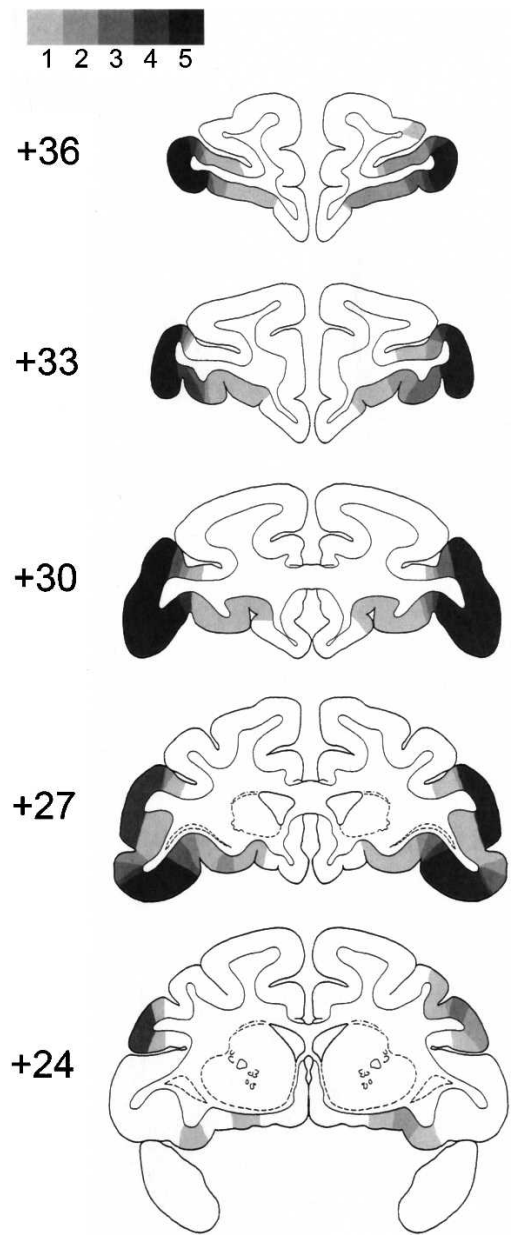

Figure 1. Areas of damage in the five monkeys with ventrolateral prefrontal lesions are plotted onto schematic coronal brain sections from a standard atlas of the rhesus monkey brain (Szwarcbart 2005). Large numerals indicate millimeters anterior to the interaural plane. The lesions are illustrated as the extent to which damage is present in one, two, three, four, or all five of the lesion cases, indicated by color going from light gray to black (the scale is represented at the top of the figure). The ventrolateral prefrontal cortex is extensively damaged in all five cases (occupying much of the inferior frontal convexity), although the lateral borders of the lesions vary from case to case.

\section{Scene learning}

Monkeys learned 20 new scene problems in each session. Each problem consisted of two foreground objects (small alphanumeric characters) presented against a complex background scene composed of a colored background, a number of colored ellipse segments, and a single large alphanumeric character (examples of scenes are shown in Fig. 3). The locations and identities of the foreground objects are fixed within each scene but vary across scenes. The monkey had to learn which of the two foreground objects in each scene was correct. In each session, each of the 20 problems was encountered once, one after the other, and then the list was presented again (in the same order) seven more times. Performance on the first trial of each problem was determined by trial and error, and learning progressed rapidly after the first trial. The performance test data comprised 10 sessions of scene learning, for a total of 200 new scenes learned. Changes in performance (percent error on each trial) between preoperative and post-operative testing were analyzed using repeated-measures ANOVA. Testing phase (preoperative vs. post-operative) and trials (repetitions of scenes within a session) were within-subject factors, and lesion group (control or ventrolateral prefrontal lesion) was a between-subjects factor.

Bilateral ablation of ventrolateral prefrontal cortex produced a significant impairment in object-in-place scene learning, with operated monkeys committing about twice as many errors post-operatively compared with control monkeys, whose error rates were similar before and after a period of rest equivalent in time to post-operative recovery for the operated group. This analysis revealed a main effect of trial, as expected, $F_{(7,49)}=478.7$, $P<0.0005$, a marginal effect of test phase, $F_{(1,7)}=5.01, P=0.06$, and a test phase by trial interaction, $F_{(7,49)}=2.88, P=0.013$. These latter two effects were driven by the performance of the ventrolateral monkeys, as they were not present when data from the controls was analyzed alone $(F s<1)$. Additionally, there were interactions of test phase with lesion group, $F_{(1,7)}=5.64$, $P=0.049$, and of test phase, trial, and lesion group, $F_{(7,49)}=2.27$, $P=0.044$. These data are plotted in Figure 4. Figure 4A shows learning curves across the eight repetitions of lists of 20 new scenes, which illustrate slower post-operative learning in the ventrolateral group. Figure 4B shows a summary measure (percentage errors on trials $2-8$ of each new list of scenes) for each monkey pre- and post-operatively, and demonstrates that each ventrolateral monkey makes more errors post-operatively, whereas performance of the control monkeys is stable. A within-subjects (preoperative vs. post-operative) comparison of the summary measure of number of errors on trials $2-8$ for the ventrolateral group revealed an identical result, $t_{(4)}=2.72, P=0.05$.

To determine whether poor post-operative performance in the ventrolateral group could be attributed to perseveration of initial incorrect responses during subsequent repetitions of each scene, the 200 scenes in each performance test were subdivided based on whether the initial response to each scene, during its first presentation in the session, was correct (1C) or wrong (1W). Correction trials (in which the incorrect object was not present) were given during the first presentation of each scene only and were not counted in the analysis. We examined the percentage of error on subsequent trials with each scene, comparing the frequency of errors with $1 \mathrm{C}$ scenes and $1 \mathrm{~W}$ scenes. This analysis revealed that monkeys with ventrolateral ablation were impaired in learning $1 \mathrm{~W}$ scenes but not $1 \mathrm{C}$ scenes, because their increase in errors post-operatively was almost entirely confined to $1 \mathrm{~W}$ scenes. Comparison of responding between preoperative and post-operative revealed expected significant main effects of trial and $1 \mathrm{C} / 1 \mathrm{~W}$, a marginal main effect of test phase (preoperative/ post-operative), and an interaction of $1 \mathrm{C} / 1 \mathrm{~W}$ with trial. Importantly, there was a significant three-way interaction of $1 \mathrm{C} / 1 \mathrm{~W}$, test phase, and group: $F_{(1,7)}=5.71, P=0.048$. This interaction was decomposed in two separate ANOVAs, which revealed a $1 \mathrm{C} / 1 \mathrm{~W}$ by test phase interaction in the ventrolateral group, $F_{(1,4)}=7.99, P=0.047$, but not in the controls, $F_{(1,3)}=0.029$, $P=0.88$. Furthermore, the effect of test phase in the ventrolateral group is not significant for $1 \mathrm{C}$ trials, $F_{(1,4)}=2.77, P=0.17$, but it is for $1 \mathrm{~W}$ trials, $F_{(1,4)}=7.93, P=0.048$. These data are plotted in Figure 5. Thus, the impairment after the ventrolateral lesion seems to stem primarily from a tendency not to correct performance based on errors made during the first run through each new list of scene problems.

Comparison of individual data points also suggests that the differential effect of ventrolateral prefrontal lesions on $1 \mathrm{C}$ versus $1 \mathrm{~W}$ trials cannot simply be ascribed to a task difficulty effect or an artifact of the scale of measurement. Consider the performance of monkeys with ventrolateral prefrontal lesions on trial 2 of $1 \mathrm{C}$ problems, and the performance on trial 3 of $1 \mathrm{~W}$ trials. Preoperatively, trial 2 of $1 \mathrm{C}$ is more difficult than trial 3 of $1 \mathrm{~W}$, in that monkeys make more errors on trial 2 of $1 \mathrm{C}$ than on trial 3 of $1 \mathrm{~W}$, and yet post-operatively, monkeys are more impaired on 


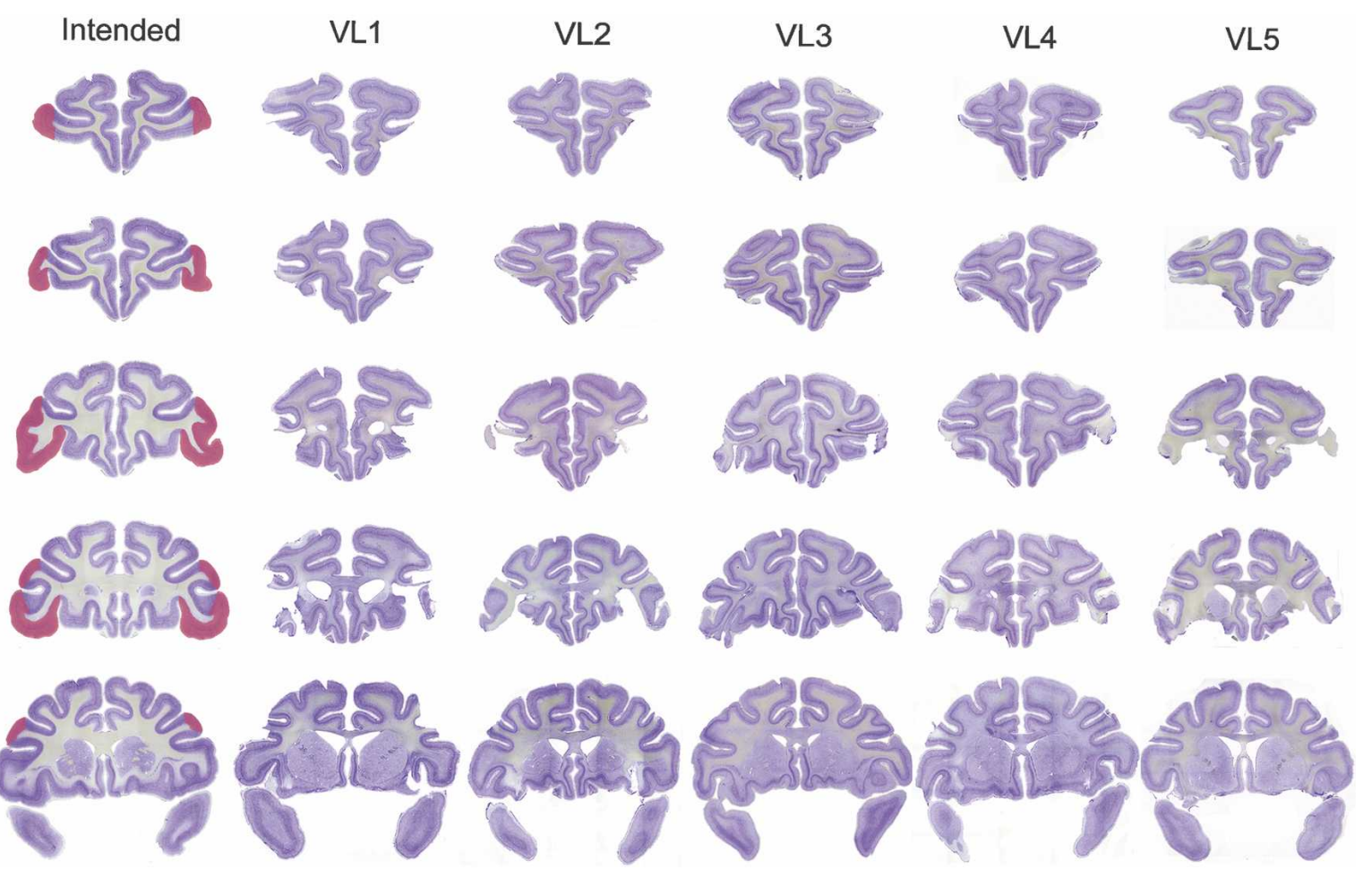

Figure 2. Lesions of VLPFC in cases VL1-VL5. The intended lesion is shown in red on coronal sections of a normal rhesus monkey in the leftmost column, at five stereotaxic levels through the prefrontal cortex, moving from rostral to caudal from the top to the bottom of the figure. Photomicrographs of brain sections from cases VL1-VL5 at corresponding stereotaxic levels are shown in the other columns.

trial 3 of $1 \mathrm{~W}$ than they are on trial 2 of $1 \mathrm{C}$. This supports the idea that the observed impairment is not simply an effect of greater difficulty, overall, of $1 \mathrm{~W}$ trials.

Similar analyses were conducted for five other groups of monkeys, whose data are reported in other publications: monkeys with bilateral ablation of orbital prefrontal cortex (Baxter et al. 2007), bilateral ablation of the entire prefrontal cortex (Browning et al. 2005), crossed unilateral lesions of frontal cortex and inferotemporal cortex (Browning et al. 2005), crossed unilateral lesions of ventrolateral prefrontal cortex and inferotemporal cortex (Wilson et al. 2007), and bilateral ablation of perirhinal cortex (Easton and Gaffan 2000). Each lesion group was considered individually in a two-way within-subjects ANOVA (lesion $\times 1 \mathrm{C} / 1 \mathrm{~W}$ ) to evaluate the significance of the lesion (test phase) $\times 1 \mathrm{C} / 1 \mathrm{~W}$ interaction, followed by focused ANOVAs to determine the effect of the lesion on $1 \mathrm{C}$ and $1 \mathrm{~W}$ trials separately as was done for the ventrolateral lesion group above. Results of these analyses are summarized in Table 1 . None of these lesion groups show the same pattern of perseverative interference in scene learning observed in the monkeys with bilateral ablation of ventrolateral prefrontal cortex in the present study, that is, a post-operative impairment in $1 \mathrm{~W}$ trials but not in 1C trials. Furthermore, this comparison between groups suggests that the lack of impairment shown by ventrolateral lesioned monkeys on 1C trials is not a result of a floor effect produced by very efficient preoperative learning in $1 \mathrm{C}$ trials. The absolute level of impairment in monkeys with bilateral orbital prefrontal lesions is similar to that of monkeys with bilateral ventrolateral prefrontal lesions, and monkeys with bilateral orbital prefrontal lesions are impaired on 1C trials in addition to $1 \mathrm{~W}$ trials.

A composite ratio measure of the post-operative impairment in $1 \mathrm{~W}$ and $1 \mathrm{C}$ trials is also presented in Table 1 . This ratio has a value of 1 when all errors scored are divided equally between $1 \mathrm{C}$ and $1 \mathrm{~W}$ trial types. The ratio is greater than 1 when more errors are scored on $1 \mathrm{~W}$ trials and less than 1 when more errors are scored on $1 \mathrm{C}$ trials. Table 1 shows that this value is close to 1 for control monkeys (who are unimpaired overall), monkeys with bilateral orbital prefrontal lesions (who are mildly impaired), and monkeys with bilateral perirhinal lesions (who are severely impaired). It is greater than 1 for monkeys whose lesions include ventrolateral prefrontal cortex, either alone or as a component of a larger prefrontal lesion or disconnection. Note that in the monkeys with larger prefrontal lesions, although the degree of impairment in $1 \mathrm{~W}$ trials is larger than $1 \mathrm{C}$ trials, the $1 \mathrm{C}$ impairment is also significant. Thus, the selective impairment on $1 \mathrm{~W}$ trials is apparently unique to bilateral lesions of ventrolateral prefrontal cortex. Comparison of the ratio measure, calculated for individual monkeys, reveals that monkeys with ventrolateral lesions have higher ratios than both unoperated controls, separate-
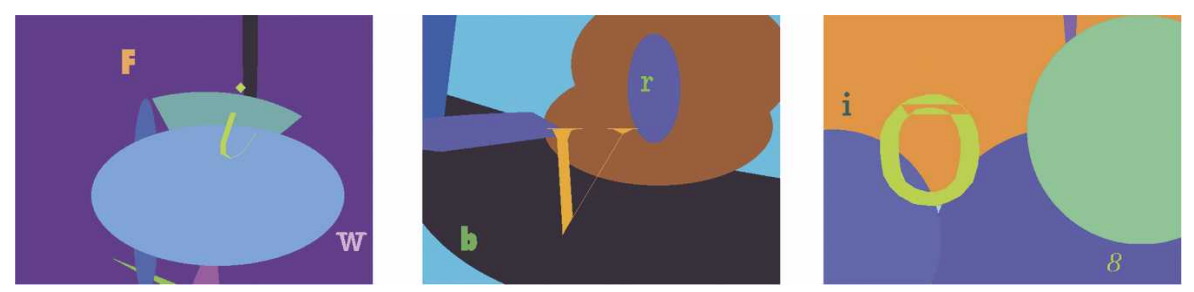

Figure 3. Three examples of scenes are shown, illustrating the background elements and the two foreground stimuli (small alphanumeric characters, e.g., $\mathrm{F}$ and $\mathrm{W}$ in the scene on the left). 

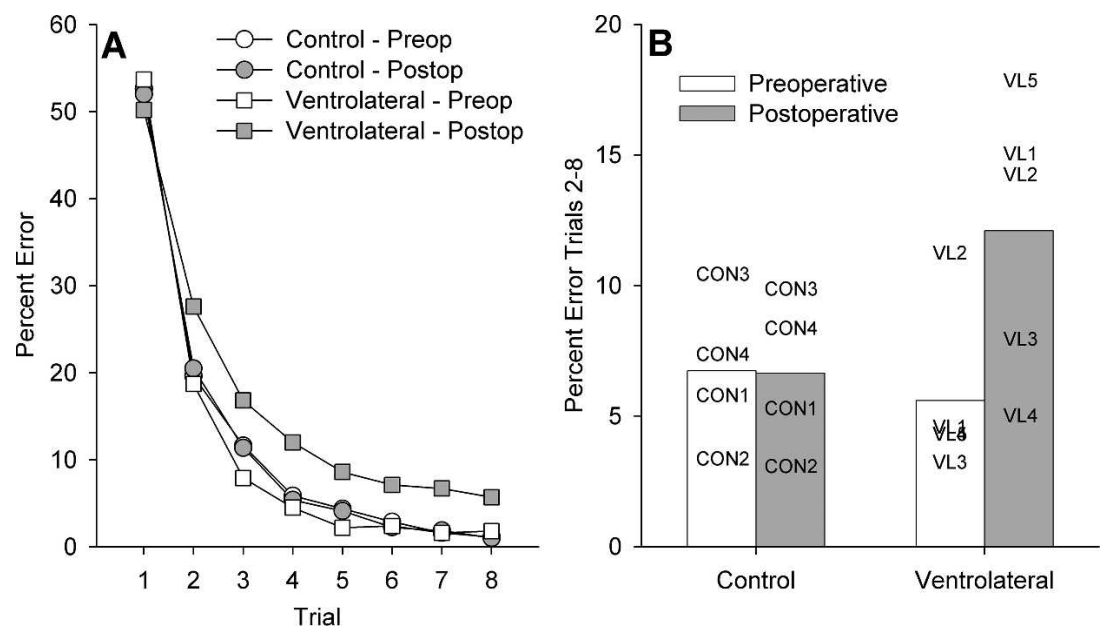

Figure 4. (A) Learning curves across trials $1-8$ for new scene learning in pre- and post-operative performance tests in control monkeys and monkeys with bilateral ventrolateral prefrontal ablation Monkeys with ventrolateral prefrontal lesions show slower learning post-operatively. (B) Individual performance on object-in-place scene learning for each monkey (CON1-CON4, controls; VL1-VL5, monkeys with bilateral ablations of ventrolateral prefrontal cortex). The measure plotted is mean performance (percentage error) across trials $2-8$ of lists of new scenes learned pre- and postoperatively. The monkeys with ventrolateral lesions are mildly impaired in scene learning postoperatively.

variance $t_{(5)}=1.99, P=0.05$ (one-tailed), and monkeys with bilateral orbital lesions, separate-variance $t_{(5.6)}=2.19, P=0.037$ (one-tailed). Individual subject data for the ratio measure are presented in Table 2 .

The same monkeys were tested on simple object-reward association learning (except VL3, who had already encountered this task preoperatively, as CON1; see Materials and Methods). We considered whether perseverative interference would also take place in this task, where monkeys learn two-choice discrimination problems between pairs of clip-art objects against a neutral background. Like scene learning, learning is by trial and error, and so the first encounter with each problem can either be correct or an error. The data from this task were analyzed in the same way, as errors/problem for $1 \mathrm{C}$ and $1 \mathrm{~W}$ problems separately, averaged across three sets of 10 problems. Monkeys made about and not for 1C trials. A separate study of selective lesions of orbital prefrontal cortex (Baxter et al. 2007) found that these lesions produced impairment on both types of trials. Larger lesions of the prefrontal cortex, which include both the ventrolateral and orbital prefrontal cortex, impair learning in both $1 \mathrm{C}$ and $1 \mathrm{~W}$ trials, although the magnitude of impairment in $1 \mathrm{~W}$ trials tended to be larger than that of $1 \mathrm{C}$ trials, consistent with the inclusion of the ventrolateral prefrontal cortex in these lesions. The new data reported in the present study suggest that perseverative responding in scene learning after lesions to prefrontal cortex may be specifically attributable to damage to the ventrolateral prefrontal cortex, rather than to the orbital prefrontal cortex. Furthermore, damage that is limited to the ventrolateral prefrontal cortex does not appear to be sufficient to produce a marked impairment in learning on trials in which the initial response is correct. Finally, this perseverative pattern of respond-
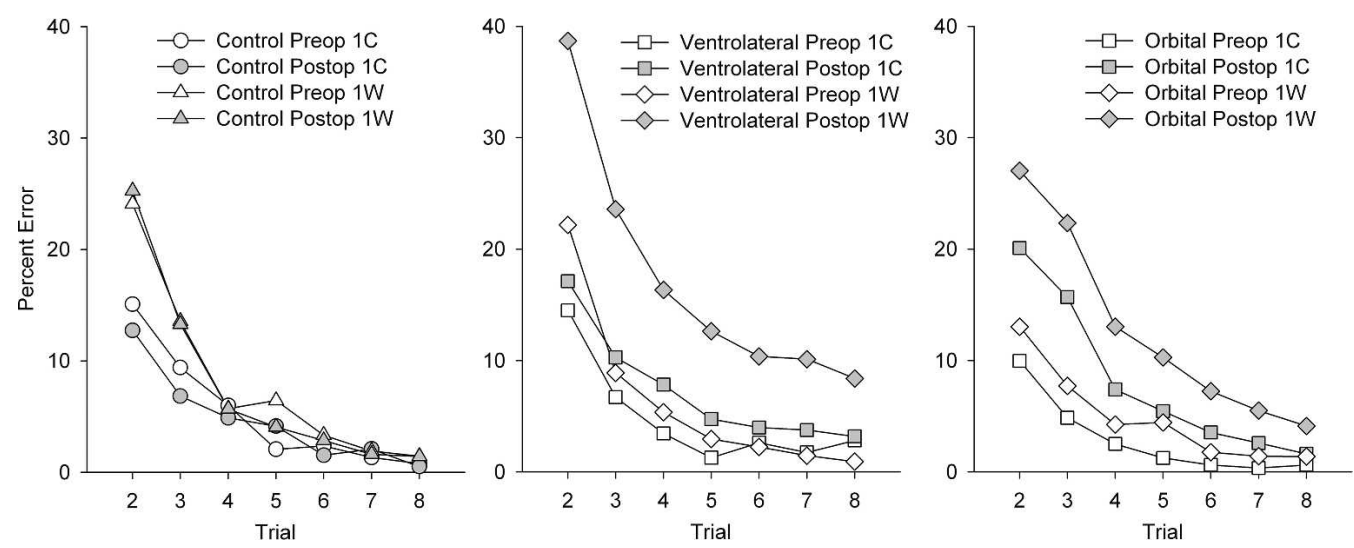

Figure 5. Performance on trials $2-8$ of learning new scene problems divided by whether the initial response to each scene was correct (1C) or wrong $(1 \mathrm{~W})$, according to each monkey's choice for each problem. (Trial 1 is omitted because, by definition, it is 0 for $1 \mathrm{C}$ scenes and 100 for $1 \mathrm{~W}$ scenes.) All monkeys make more errors in learning scenes to which their initial response is incorrect. The performance of control monkeys is indistinguishable between their two performance tests (before and after a rest period equivalent to the post-operative recovery time of monkeys that receive surgery). Ablation of ventrolateral prefrontal cortex does not significantly increase the errors monkeys make in learning $1 \mathrm{C}$ scenes, but it substantially impairs learning of $1 \mathrm{~W}$ scenes. Data from monkeys with bilateral ablation of orbital prefrontal cortex are shown for comparison (Baxter et al. 2007). These lesions produce a similar overall deficit in scene learning in terms of total errors on trials $2-8$, but this effect is due to a mild impairment in learning in both $1 \mathrm{C}$ and 1W trials. Left and right panels were replotted with permission from the Society for Neuroscience @ 2007, Figure 5 of Baxter et al. 2007. 
Table 1. Comparison of scene learning impairments after different cortical lesions

\begin{tabular}{|c|c|c|c|c|c|c|c|c|c|c|c|c|c|}
\hline \multirow[b]{2}{*}{ Lesion } & \multicolumn{3}{|c|}{ Preoperative (\% error) } & \multicolumn{3}{|c|}{ Post-operative (\% error) } & \multicolumn{5}{|c|}{ Effects } & \multirow[b]{2}{*}{ Ratio $^{a}$} & \multirow[b]{2}{*}{ Reference } \\
\hline & Overall & 1C & $1 \mathrm{~W}$ & Overall & 1C & $1 \mathrm{~W}$ & Lesion & Lesion & $\times 1 C / 1 W$ & 1C & $1 \mathrm{~W}$ & & \\
\hline Control & 6.73 & 5.29 & 8.00 & 6.65 & 5.11 & 7.97 & - & & - & - & - & 0.992 & This study ${ }^{b}$ \\
\hline Ventrolateral PFC & 5.59 & 4.74 & 6.29 & 12.07 & 7.28 & 17.16 & * & & * & - & * & 1.698 & This study \\
\hline Orbital PFC & 4.02 & 2.89 & 5.27 & 10.73 & 8.35 & 13.27 & ** & & * & ** & ** & 0.880 & Baxter et al. 2007 \\
\hline Bilateral PFC & 16.68 & 12.88 & 20.75 & 46.45 & 25.05 & 68.55 & ** & & ** & * & ** & 1.726 & Browning et al. 2005 \\
\hline $\mathrm{FL} \times \mathrm{IT}$ & 6.50 & 5.47 & 7.43 & 29.67 & 19.70 & 40.43 & ** & & * & * & ** & 1.550 & Browning et al. 2005 \\
\hline VIPFC $\times$ IT & 12.48 & 9.80 & 15.26 & 29.07 & 20.04 & 37.80 & ** & & - & * & * & 1.438 & Wilson et al. 2007 \\
\hline PRh & 8.97 & 4.67 & 13.13 & 38.27 & 22.33 & 56.07 & * & & ** & * & * & 0.944 & Easton and Gaffan 2000 \\
\hline
\end{tabular}

$1 \mathrm{C}$, first trial correct; $1 \mathrm{~W}$, first trial wrong; $\mathrm{FL} \times \mathrm{IT}$, frontal lobe/inferotemporal cortex disconnection; PFC, prefrontal cortex; PRh, bilateral lesion of perirhinal cortex; and VIPFC $\times$ IT, ventrolateral prefrontal/inferotemporal cortex disconnection.

${ }^{\mathrm{a}}$ Ratio $=($ post-operative $1 \mathrm{~W} /$ preoperative $1 \mathrm{~W}) /($ post-operative $1 \mathrm{C} /$ preoperative $1 \mathrm{C})$. The value shown in the table is the mean ratio for all the monkeys in each group $(n=3-5)$, not the ratio of the mean percentage error for each condition.

${ }^{\mathrm{b}}$ Control data also presented and used for comparison in other papers (Baxter et al. 2007; Mitchell et al. 2007).

${ }^{*} P<0.05 ;{ }^{* *} P<0.01$.

ing is not seen in another associative learning task (object-reward association learning) that requires an intact prefrontal cortex but does not require frontal-inferotemporal interaction (Parker and Gaffan 1998). Thus, it may reflect a specific contribution of ventrolateral prefrontal cortex to episodic memory.

The present results are consistent with an early report of perseverative behavior in reversal learning after selective lesions of the inferior frontal convexity (Iversen and Mishkin 1970). However, they are somewhat surprising in light of the association of perseverative behavior with damage to the orbital prefrontal cortex (e.g., Jones and Mishkin 1972). It must be noted that many studies of "orbital" prefrontal cortex include damage to the prefrontal cortex termed ventrolateral in the present study and in other investigations (e.g., Rushworth et al. 1997; see also Petrides and Pandya 2002). Recent investigations of discrete lesions of the orbital prefrontal cortex have reported deficits in reversal learning that are not perseverative in nature (Izquierdo et al. 2004), consistent with the present findings. It may be difficult in other settings to distinguish errors that are perseverative in nature from errors that reflect failures in acquiring new stimulus-reward associations, for example, in extinction performance (Izquierdo and Murray 2005).

It is important to compare the present results with the study of crossed unilateral lesions of the ventrolateral prefrontal cortex (made with neurotoxin injections) and inferotemporal cortex (Wilson et al. 2007), which reported impairments in scene learning that were more severe than those found for bilateral ablations of ventrolateral prefrontal cortex in the present study. When these results are compared, two of the three lesion cases in the study of disconnection lesions had neurotoxin injections into the orbital surface in addition to lesions of the ventrolateral prefrontal cortex, although the injections of the orbital surface were observed only to produce small foci of cell loss rather than frank lesions of orbital prefrontal cortex. Thus, the severity of their impairments may reflect the combination of ventrolateral damage and partial lesions of the orbital cortex. This may also explain the fact that monkeys with the neurotoxic disconnection lesions were significantly impaired in both $1 \mathrm{C}$ and $1 \mathrm{~W}$ trials, although their $1 \mathrm{~W}$ impairment was more severe.

The impairment after bilateral ablations of ventrolateral prefrontal cortex is not larger in magnitude than that observed after neurotoxic ventrolateral lesions (crossed with inferotemporal ablation). This suggests that the deleterious effect of bilateral ventrolateral ablation in scene learning probably cannot be ascribed to the interruption of fibers of passage through the ablated region of cortex, an explanation suggested for some previous effects of ablations of this region (Mishkin and Manning 1978).

The comparison of scene learning impairments after lesions of orbital prefrontal cortex (Baxter et al. 2007) and ventrolateral prefrontal cortex (present study) is instructive in terms of understanding different contributions of these two areas of prefrontal cortex to episodic learning and memory. In our earlier study of orbital prefrontal lesions, we theorized that the mild effect of these lesions on scene learning reflected a contribution of this

Table 2. Individual subject data for object-in-place scene learning pre- and post-operatively, broken down by 1C (initial response correct) and $1 \mathrm{~W}$ (initial response wrong) scenes

\begin{tabular}{|c|c|c|c|c|c|c|c|c|}
\hline \multirow[b]{2}{*}{ Group } & \multirow[b]{2}{*}{ Case } & \multicolumn{3}{|c|}{ Preoperative (\% error) } & \multicolumn{3}{|c|}{ Post-operative (\% error) } & \multirow[b]{2}{*}{ Ratio } \\
\hline & & Overall & $1 \mathrm{C}$ & $1 \mathrm{~W}$ & Overall & $1 C$ & $1 \mathrm{~W}$ & \\
\hline \multirow[t]{4}{*}{ Control } & CON1 & 5.79 & 2.61 & 8.52 & 5.29 & 3.12 & 6.95 & 0.683 \\
\hline & CON2 & 3.36 & 3.23 & 3.47 & 3.07 & 3.05 & 3.09 & 0.942 \\
\hline & CON3 & 10.40 & 8.87 & 11.84 & 9.86 & 7.22 & 12.15 & 1.261 \\
\hline & CON4 & 7.36 & 6.47 & 8.16 & 8.36 & 7.07 & 9.67 & 1.083 \\
\hline \multirow[t]{5}{*}{ Ventrolateral PFC } & VL1 & 4.64 & 3.74 & 5.30 & 15.10 & 6.25 & 26.46 & 2.991 \\
\hline & VL2 & 11.30 & 10.71 & 11.77 & 14.30 & 10.64 & 17.27 & 1.478 \\
\hline & VL3 & 3.29 & 1.62 & 4.59 & 8.00 & 3.91 & 11.70 & 1.058 \\
\hline & VL4 & 4.36 & 3.79 & 4.90 & 5.07 & 3.57 & 6.57 & 1.423 \\
\hline & VL5 & 4.36 & 3.82 & 4.91 & 17.90 & 12.02 & 23.81 & 1.542 \\
\hline \multirow[t]{3}{*}{ Orbital PFC } & ORB1 & 3.64 & 1.51 & 5.95 & 10.60 & 6.72 & 14.58 & 0.550 \\
\hline & ORB2 & 2.00 & 1.81 & 2.18 & 9.50 & 8.12 & 10.93 & 1.116 \\
\hline & ORB3 & 6.43 & 5.34 & 7.68 & 12.10 & 10.20 & 14.29 & 0.973 \\
\hline
\end{tabular}

${ }^{\text {aRatio }}=$ (post-operative $1 \mathrm{~W} /$ preoperative $1 \mathrm{~W}$ )/(post-operative $1 \mathrm{C} /$ preoperative $1 \mathrm{C}$ ) as in Table 1.

PFC, prefrontal cortex. 
region to noticing novel information and facilitating its encoding in inferotemporal cortex (Frey and Petrides 2002) or to strategic processes used to optimize encoding of information in memory (Kao et al. 2005). Ventrolateral prefrontal cortex has been hypothesized to be involved in multiple cognitive control processes in episodic memory (Badre et al. 2005; Badre and Wagner 2007). In particular, this region may contribute importantly to the resolution of proactive interference in memory (Badre and Wagner 2005). This is consistent with the effect of ventrolateral prefrontal lesions on scene learning in the present study, in which a selective deficit in $1 \mathrm{~W}$ trials can be interpreted as a failure to resolve interference between the initial trial-and-error response to the scene with the feedback from that trial that the initial choice is incorrect. Another possibility, not exclusive of the foregoing one, is that ventrolateral prefrontal cortex is specifically engaged when a response to negative feedback must be planned (Monchi et al. 2001). The disruption of processing in both orbital and ventrolateral prefrontal cortex following bilateral prefrontal damage or frontal-inferotemporal disconnection (Browning et al. 2005) results in severe deficits in episodic memory, whereas the disruption of one or the other produces comparatively mild impairments. Future experiments may test specifically the extent to which these processes may interact with one another, as well as by parametrically manipulating the amount of proactive interference in memory by altering parameters of the scene learning task, for example, by increasing the similarity between elements of the background scenes. This may increase the extent to which the macaque monkey model of episodic memory may be useful for translating between studies of the neurobiological mechanisms that underlie this ability in monkeys with neuropsychological and neuroimaging studies of episodic memory in humans.

\section{Materials and Methods}

\section{Subjects}

Eight rhesus monkeys (Macaca mulatta), six male (CON1/VL3, CON2, CON4, VL1, VL2, VL5) and two female (CON3, VL4), $3.33-7.44 \mathrm{~kg}$ (22- to 51-mo-old) at the beginning of behavioral training, participated in this study. The monkeys were housed socially in troops, separated by sex, in indoor enclosures attached to standard caging. Water was always available ad libitum in the home enclosure; each monkey's daily food ration was delivered in the test box and was supplemented with fruit and forage mix in the home enclosure.

Object-in-place scene learning was tested alongside performance in a strategy implementation task (Gaffan et al. 2002). Results from the strategy implementation task, and subsequent post-operative testing in discrimination learning, are described in a separate publication. The critical test data for scene learning came from pre- and post-operative performance tests, which were identical for all monkeys, and consisted of $2 \mathrm{~d}$ of strategy implementation testing alternating with $2 \mathrm{~d}$ of scene learning for a total of 12 sessions of each task, the last 10 of which constituted the data for the performance test (see also Baxter et al. 2007). Five of the monkeys (VL1, VL2, CON1/VL3, VL4, VL5) received bilateral ablations of ventrolateral prefrontal cortex. Three monkeys (CON2, CON3, CON4) remained as unoperated controls. One monkey (CON1/VL3) completed the entire sequence of pre- and post-operative testing as an unoperated control and then received a further performance test on scene learning and strategy implementation, a ventrolateral prefrontal lesion, and then a post-operative performance test on scene learning and strategy implementation. For purposes of data analysis, he is treated as two separate cases, and is designated CON1 when his data from his first performance test are discussed and as VL3 when his data related to his ventrolateral lesion are discussed (including his second preoperative performance test).

\section{Object-in-place scene learning}

The object-in-place scene learning task was adapted from the method of Gaffan (1994). This task employed artificially constructed background scenes that occupied the whole area of the display screen. The background scenes were generated by an algorithm based on a random number generator. Each scene was unique in that it varied in several randomly selected attributes, including (1) the background color of the screen; (2) the location of ellipses on the screen; (3) the color, size, and orientation of ellipse segments; (4) the typographic character, clearly distinct in size from the foreground objects; and (5) the color of the typographic character. All the colors were assigned with the constraint that the foreground objects should be visible (i.e., there was a minimum separation in color space between the colors of a foreground object and the color of any element of its local background). Two background objects, small randomly chosen and colored typographic characters, were placed within each scene. In each scene, one of the two foreground objects was the correct one for the monkey to touch (rewarded) and the other was incorrect (unrewarded). The locations and identities of the foreground objects were fixed within each scene but varied between scenes. Because these scenes were randomly generated, an infinite number of unique scenes could be presented. (For examples of stimuli, see Gaffan 1994; Browning et al. 2005; Fig. 3). After each monkey learned to touch single foreground objects against a black background, additional scene elements were introduced in shaping programs until the monkey reliably touched the foreground object when presented with a new scene. Problems were then introduced with two foreground objects (one correct and one incorrect, as described above), and the number of scenes given in each session was gradually increased, based on each monkey's performance. Training continued until performance was stable (for all eight monkeys, mean of 58.1 sessions, range 30-111).

In the final version of the task, 20 new scenes were presented in each session; the list of 20 scenes was repeated eight times. Each trial began with the presentation of a scene problem on the screen (a background scene containing two foreground objects). A touch to the correct object caused the object to flash for $2.4 \mathrm{sec}$, and then the screen blanked and a reward pellet (190 $\mathrm{mg}$; P.J. Noyes) was delivered, followed by a 5-sec intertrial interval. A touch to the incorrect object caused the screen to blank immediately, followed by a 20 -sec intertrial interval. Touches anywhere else in the scene caused the screen to blank and the trial was repeated, following a 20 -sec intertrial interval. For the first repetition of the list of scenes only, incorrect responses were followed by a correction trial in which the scene was re-presented with only the correct object present. The subsequent seven repetitions of the list of scenes did not contain correction trials, and the scenes were presented in the same order in which they were encountered in the first run through the list. Monkeys learned which object in each scene was correct by trial and error, generally very rapidly during the first run through the list, because error rates were very low during the second run through the list (9\%-21.5\%; chance is 50\%). When the monkey completed the final trial of a session, the lunchbox opened and the monkey received the large food reward. If the final trial was incorrect, a correction trial was given so that the monkey only ever received the large food reward following a correct response. The dependent measure was the number of errors (initial touches of the incorrect foreground object) in each presentation of the list of 20 scenes.

\section{Surgery}

Neurosurgical procedures were performed in a dedicated operating theater under aseptic conditions. Each operated monkey's neurosurgical procedure consisted of a bilateral ablation of the ventrolateral prefrontal cortex. In cases VL2-VL5, steroids (methylprednisolone, $20 \mathrm{mg} / \mathrm{kg}$ ) were given intramuscularly (i.m.) the night before surgery, and three doses were given 4-6 $\mathrm{h}$ apart (intravenously [i.v.] or i.m.) on the day of surgery, to protect against intraoperative edema and post-operative inflammation. 
Case VL1 received intravenous dexamethasone $(2 \mathrm{mg} / \mathrm{kg})$ i.v. once during the surgery only. Each monkey was sedated on the morning of surgery with both ketamine $(10 \mathrm{mg} / \mathrm{kg})$ and xylazine $(0.5 \mathrm{mg} / \mathrm{kg})$, i.m. Once sedated, the monkey was given atropine $(0.05 \mathrm{mg} / \mathrm{kg})$ to reduce secretions, antibiotic (amoxicillin, 8.75 $\mathrm{mg} / \mathrm{kg}$ ) for prophylaxis of infection, opioid (buprenorphine 0.01 $\mathrm{mg} / \mathrm{kg}$ i.v., repeated twice at 4-6 h intervals on the day of surgery, i.v. or i.m.) and nonsteroidal anti-inflammatory (either meloxicam, $0.2 \mathrm{mg} / \mathrm{kg}$, i.v. or carprofen, $4 \mathrm{mg} / \mathrm{kg}$, i.m.) agents for analgesia, and an $\mathrm{H} 2$ receptor antagonist (ranitidine, $1 \mathrm{mg} / \mathrm{kg}$, i.v.) to protect against gastric ulceration as a side-effect of the combination of steroid and nonsteroidal anti-inflammatory treatment. The head was shaved and an intravenous cannula put in place for intraoperative delivery of fluids (warmed sterile saline drip, 5 $\mathrm{mL} / \mathrm{h} / \mathrm{kg}$ ). The monkey was moved into the operating theater, intubated, placed on isoflurane (VL1, VL3, VL4, 1-2.5\%, to effect, in $100 \%$ oxygen) or sevoflurane (VL2 and VL5, $2.25-4.5 \%$, to effect, in $100 \%$ oxygen) anesthesia, and then mechanically ventilated. Adjustable heating blankets allowed maintenance of normal body temperature during surgery. Heart rate, oxygen saturation of hemoglobin, mean arterial blood pressure, end tidal $\mathrm{CO}_{2}$, body temperature, and respiration rate were monitored continuously throughout surgery.

The monkey was placed in a head-holder and the head cleaned with alternating antimicrobial scrub and alcohol and draped to allow a midline incision. The skin and underlying galea were opened in layers. The temporal muscles were retracted as necessary to expose the skull surface over the intended lesion site. A bone flap was turned over the frontal lobes, and the craniotomy was extended with rongeurs as necessary. The dura was cut and reflected over the frontal lobes. The ventrolateral prefrontal cortex was removed bilaterally extending from the ventral lip of the principal sulcus to the fundus of the lateral orbital sulcus. The anterior limit was a line joining the anterior tips of the principal and lateral orbital sulci. The posterior limit was a line joining the posterior tip of the principal sulcus and the anterior tip of the inferior limb of the arcuate sulcus, then extending from the tip of the arcuate sulcus to the posterior tip of the lateral orbital sulcus. All of the cortex was removed within these limits (cf. Rushworth et al. 1997). Cortical tissue was removed by subpial aspiration using a small-gauge sucker insulated everywhere except at the tip; electrocautery was applied to remove the pia mater and control bleeding encountered during the ablation.

When the lesion was complete, the dura was sewn over the lesion site, the bone flap replaced and held with loose sutures, and the skin and galea were closed in layers. The monkey was removed from the head-holder and anesthesia discontinued. The monkey was extubated when a swallowing reflex was observed, returned to the home cage, and monitored continuously until normal posture was regained (usually within $10 \mathrm{~min}$ ). Nonsteroidal anti-inflammatory analgesic (meloxicam, $0.2 \mathrm{mg} / \mathrm{kg}$, oral) and antibiotic (amoxicillin, $8.75 \mathrm{mg} / \mathrm{kg}$, oral) treatment continued following surgery, in consultation with veterinary staff, for 4-5 d. Operated monkeys rejoined their social groups as soon as practicable after surgery, usually within $3 \mathrm{~d}$ of the operation.

\section{Acknowledgments}

We thank M. Brown, G. Daubney, K. Murphy, and S. Mygdal for technical support, and D. Gaffan for performing the surgeries. Research supported by the Wellcome Trust (M.G.B.) and the Medical Research Council (program grant to D. Gaffan).

\section{References}

Aggleton, J.P., McMackin, D., Carpenter, K., Hornak, J., Kapur, N., Halpin, S., Wiles, C.M., Kamel, H., Brennan, P., Carton, S., et al 2000. Differential cognitive effects of colloid cysts in the third ventricle that spare or compromise the fornix. Brain 123: 800-815.

Badre, D. and Wagner, A.D. 2005. Frontal lobe mechanisms that resolve proactive interference. Cereb. Cortex 15: 2003-2012.

Badre, D. and Wagner, A.D. 2007. Left ventrolateral prefrontal cortex and the cognitive control of memory. Neuropsychologia 45: $2883-2901$.
Badre, D., Poldrack, R.A., Pare-Blagoev, E.J., Insler, R.Z., and Wagner, A.D. 2005. Dissociable controlled retrieval and generalized selection mechanisms in ventrolateral prefrontal cortex. Neuron 47: 907-918.

Baxter, M.G., Gaffan, D., Kyriazis, D.A., and Mitchell, A.S. 2007. Orbital prefrontal cortex is required for object-in-place scene memory but not performance of a strategy implementation task. J. Neurosci. 27: 11327-11333.

Browning, P.G.F., Easton, A., Buckley, M.J., and Gaffan, D. 2005. The role of prefrontal cortex in object-in-place learning in monkeys. Eur. J. Neurosci. 22: 3281-3291.

Easton, A. and Gaffan, D. 2000. Comparison of perirhinal cortex ablation and crossed unilateral lesions of the medial forebrain bundle from the inferior temporal cortex in the rhesus monkey: Effects on learning and retrieval. Behav. Neurosci. 114: 1041-1057.

Frey, S. and Petrides, M. 2002. Orbitofrontal cortex and memory formation. Neuron 36: 171-176.

Gaffan, D. 1994. Scene-specific memory for objects: A model of episodic memory impairment in monkeys with fornix transection. J. Cogn. Neurosci. 6: $305-320$.

Gaffan, D., Easton, A., and Parker, A. 2002. Interaction of inferior temporal cortex with frontal cortex and basal forebrain: Double dissociation in strategy implementation and associative learning. $J$. Neurosci. 22: 7288-7296.

Goldman-Rakic, P.S. 1996. The prefrontal landscape: Implications of functional architecture for understanding human mentation and the central executive. Philos. Trans. R. Soc. Lond. B Biol. Sci. 351: $1445-1453$.

Iversen, S.D. and Mishkin, M. 1970. Perseverative interference in monkeys following selective lesions of the inferior prefrontal convexity. Exp. Brain Res. 11: 376-386.

Izquierdo, A. and Murray, E.A. 2005. Opposing effects of amygdala and orbital prefrontal cortex lesions on the extinction of instrumental responding in macaque monkeys. Eur. J. Neurosci. 22: 2341-2346.

Izquierdo, A., Suda, R.K., and Murray, E.A. 2004. Bilateral orbital prefrontal cortex lesions in rhesus monkeys disrupt choices guided by both reward value and reward contingency. J. Neurosci. 24: 7540-7548.

Jones, B. and Mishkin, M. 1972. Limbic lesions and the problem of stimulus-reinforcement associations. Exp. Neurol. 36: 362-377.

Kao, Y.C., Davis, E.S., and Gabrieli, J.D. 2005. Neural correlates of actual and predicted memory formation. Nat. Neurosci. 8: 1776-1783.

Miller, E.K. 2000. The prefrontal cortex and cognitive control. Nat. Rev. Neurosci. 1: 59-65.

Mishkin, M. and Manning, F.J. 1978. Non-spatial memory after selective prefrontal lesions in monkeys. Brain Res. 143: 313-323.

Mitchell, A.S., Baxter, M.G., and Gaffan, D. 2007. Dissociable performance on scene learning and strategy implementation after lesions to magnocellular mediodorsal thalamic nucleus. J. Neurosci. 27: 11888-11895.

Monchi, O., Petrides, M., Petre, V., Worsley, K., and Dagher, A. 2001. Wisconsin Card Sorting revisited: Distinct neural circuits participating in different stages of the task identified by event-related functional magnetic resonance imaging. J. Neurosci. 21: 7733-7741.

Parker, A. and Gaffan, D. 1998. Memory after frontal/temporal disconnection in monkeys: conditional and non-conditional tasks, unilateral and bilateral frontal lesions. Neuropsychologia 36: 259-271.

Passingham, R.E. 1993. The frontal lobes and voluntary action. Oxford University Press, Oxford.

Petrides, M. and Pandya, D.N. 2002. Comparative cytoarchitectonic analysis of the human and the macaque ventrolateral prefrontal cortex and corticocortical connection patterns in the monkey. Eur. J. Neurosci. 16: 291-310.

Petrides, M., Alivisatos, B., and Frey, S. 2002. Differential activation of the human orbital, mid-ventrolateral, and mid-dorsolateral prefrontal cortex during the processing of visual stimuli. Proc. Natl. Acad. Sci. 99: 5649-5654.

Rushworth, M.F., Nixon, P.D., Eacott, M.J., and Passingham, R.E. 1997. Ventral prefrontal cortex is not essential for working memory. J. Neurosci. 17: 4829-4838.

Szwarcbart, M. 2005. Stereotaxic brain atlas of rhesus monkey, Red. http://neuron.nimh.nih.gov/Red.zip.

Wagner, A.D., Pare-Blagoev, E.J., Clark, J., and Poldrack, R.A. 2001. Recovering meaning: Left prefrontal cortex guides controlled semantic retrieval. Neuron 31: 329-338.

Wilson, C.R.E., Gaffan, D., Mitchell, A.S., and Baxter, M.G. 2007. Neurotoxic lesions of ventrolateral prefrontal cortex impair object-in-place scene memory. Eur. J. Neurosci. 25: 2514-2522.

Received October 12, 2007; accepted in revised form December 29, 2007. 


\section{Erratum}

Learning \& Memory 15: 126-132 (2008)

Perseverative interference with object-in-place scene learning in rhesus monkeys with bilateral ablation of ventrolateral prefrontal cortex

Mark G. Baxter, Philip G.F. Browning, and Anna S. Mitchell

The Abstract in this paper's full-text view online has been adjusted to reflect the correct Abstract for this paper. 


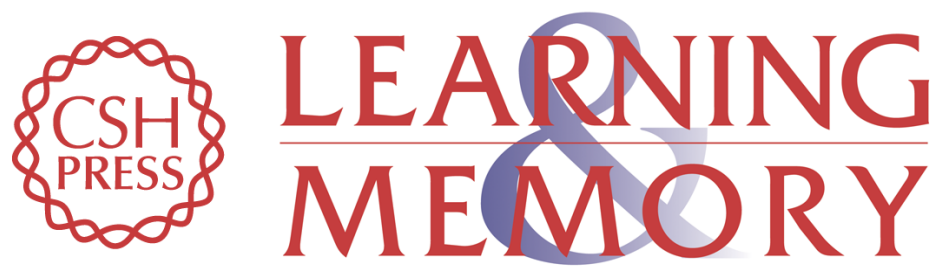

\section{Perseverative interference with object-in-place scene learning in rhesus monkeys with bilateral ablation of ventrolateral prefrontal cortex}

Mark G. Baxter, Philip G.F. Browning and Anna S. Mitchell

Learn. Mem. 2008, 15:

Access the most recent version at doi:10.1101//m.804508

Related Content Erratum

Learn. Mem. April , 2008 15: 189

References This article cites 26 articles, 7 of which can be accessed free at: http://learnmem.cshlp.org/content/15/3/126.full.html\#ref-list-1

Articles cited in:

http://learnmem.cshlp.org/content/15/3/126.full.html\#related-urls

License Freely available online through the Learning \& Memory Open Access option.

Email Alerting Receive free email alerts when new articles cite this article - sign up in the box at the Service top right corner of the article or click here. 